\title{
Increased risk of deep vein thrombosis in end-stage renal disease patients
}

\author{
Hsueh-Yi Lu ${ }^{1}$ and Kuang-Ming Liao ${ }^{2^{*}}$ (D)
}

\begin{abstract}
Background: Previous studies have shown that chronic kidney disease increases the risk of deep vein thrombosis (DVT). DVT is the risk of pulmonary embolism among persons with end-stage renal disease (ESRD). Information on the incidence of DVT in ESRD is limited, and no studies have been conducted in the Asian population. The aim of our study was to investigate the incidence of DVT in Asian ESRD patients by comparing with the non-ESRD patients and to identify the associated risk factors.

Methods: This study retrieved patients who were diagnosed with ESRD (ICD-9-CM codes 585 or 586) between January 1, 2004, and December 31, 2010, from the National Health Insurance Research Database in Taiwan. All ESRD patients had received a catastrophic illness card from the Ministry of Health and Welfare in Taiwan, with the major illness identified as ESRD. Patients who had DVT before the index date or who had incomplete records were excluded from the analysis. A total of 4865 ESRD patients were enrolled. There are 3564 ESRD patients included after exclusion of patients with previous DVT and patients with incomplete records. The control subjects were randomly selected as the patients without ESRD by matching study subjects according to age ( \pm 3 years), gender, and the year of admission at a 2:1 ratio from the same dataset.

Results: The incidence rate of DVT was substantially higher in the ESRD group than in the without-ESRD group (20.9 vs. 1.46 per 10^4 person-years). The adjusted hazard ratio (aHR 13.92; 95\% Cl 9.25-20.95) of DVT for the ESRD patients was 13.92 times that for the non-ESRD patients. ESRD patients older than 50 years had a higher risk of DVT (aHR 1.65; 95\% Cl 1.13-2.40; $P=0.01$ ). Hyperlipidemia was significantly associated with an increased risk of DVT (aHR 1.73; 95\% Cl 1.08-2.78; $P=0.02$ ). ESRD patients with three or more comorbidities were substantially more likely to have DVT (aHR 1.45; 95\% Cl 1.03-2.03; $P=0.03$ ).

Conclusions: ESRD patients had a higher risk of DVT than non-ESRD patients. Among the ESRD patients, being older than 50 years and having dyslipidemia increased the risk of DVT.
\end{abstract}

Keywords: Deep vein thrombosis, End-stage renal disease, Incidence

\section{Background}

The epidemiology of deep vein thrombosis (DVT) has been studied in the general population [1-3]. .End-stage renal disease is associated with a 2.3 -fold increased risk of DVT compared with the general population [4]. The rate of DVT occurring 1.5 to 3 years after transplantation was 2.9 episodes/1000 person-years. Patients with severe renal function impairment at the end of the first year after renal transplantation increased risk for DVT [5]. Otherwise, there is high risk of DVT recurrence in

\footnotetext{
* Correspondence: abc8870@yahoo.com.tw

${ }^{2}$ Department of Internal Medicine, Chi Mei Medical Center, Chiali, Taiwan

Full list of author information is available at the end of the article
}

patients developing a first episode of DVT after renal transplant [6]. The incidence of DVT ranges from 48 to 182 per 100,000 observation-years and increases with age. The risk factors for DVT include old age, surgery, cancer, hospitalization, stasis, immobilization, obesity, trauma, exogenous hormones, pregnancy and inherited thrombophilia [7]. Chronic kidney disease (CKD) is associated with increased procoagulants, including cystatin $\mathrm{C}, \mathrm{C}$-reactive protein, interleukin-6, tumor necrosis factor- $\alpha$ soluble receptor 1, intercellular adhesion molecule-1, fibrinogen, and factor VIII [8]. Previous studies have shown that CKD increases the risk of DVT, and its epidemiology and clinical implications have been reported [4, 9-14]. The

(c) The Author(s). 2018 Open Access This article is distributed under the terms of the Creative Commons Attribution 4.0 International License (http://creativecommons.org/licenses/by/4.0/), which permits unrestricted use, distribution, and 
prevalence of pulmonary thromboembolism has been reported at autopsy [15]. DVT is considered an uncommon disease in patients with end-stage renal disease (ESRD) [16]. A prospective study conducted in a university-affiliated intensive care unit found that ESRD was a risk factor for DVT (hazard ratio (HR) 3.7, 95\% confidence interval (CI) 1.2-11.1). To our knowledge, studies examining DVT and ESRD have been limited. The aim of our study was to assess the incidence of DVT in Asian ESRD patients by comparing with the non-ESRD patients and to identify the associated risk factors for DVT within ESRD patients.

\section{Methods}

\section{Ethics statement}

This study was approved by the Institutional Review Board (IRB) of the Chi Mei Medical Center, Taiwan (10609-E02). The anonymity of the selected subjects was preserved by encrypting the original identification information before further analysis. Informed consent was waived by the approving IRB.

\section{Data sources}

The National Health Insurance (NHI) is a public singlepayer insurance program that has provided nationwide coverage in Taiwan since 1995. It covers approximately 99\% of the population and contracts with $97 \%$ of healthcare providers. The NHI Research Database (NHIRD), which is authorized and released for research purposes, contains all the inpatient and outpatient registration and claims data of the NHI program. The data include patients' demographic characteristics, disease-diagnostic and surgery-operation codes (based on the International Classification of Diseases, Ninth Revision, Clinical Modification [ICD-9-CM]), prescription records, and medical expenditures. The NHIRD is one of the largest administrative health care databases in the world. This study used a longitudinally linked NHIRD dataset, which consists of a cohort of one million randomly selected enrollees traced retrospectively from 1996 to 2010. No statistically significant differences were found in the age, sex, and health care cost distributions of the selected subjects.

\section{Patients}

This study utilized data from patients who were diagnosed with ESRD (ICD-9-CM codes 585 or 586) between January 1, 2004, and December 31, 2010. In Taiwan, patients with ESRD requiring dialysis can apply for a catastrophic illness card and free of any charge. The aim of our study was to assess the incidence of DVT in Asian ESRD patients. Before patients had received a catastrophic illness card of ESRD from the Ministry of Health and Welfare in Taiwan, patients may include the ICD-9-CM codes 585 and 586. We linked the Catastrophic Illness Patient
Database file to get the diagnosis of ESRD. Because each individual registered in the catastrophic illnesses database is exempted from any co-payment for treatment, the process for evaluating applicants' eligibility for this registry is strict and comprehensive. For the case of ESRD, the catastrophic illness certification was issued by a nephrologist and confirmed by another nephrologist. Under this condition, the possibility of ESRD identified in our study being true positive is considerably high. The application date of the catastrophic illness card was designated the index date as a starting time point to estimate the risk of DVT. Patients who had DVT before the index date or who had incomplete records were excluded from the analysis. A total of 4865 qualified patients who had ESRD were preliminarily identified before the exclusion filtering (Fig. 1).

\section{Outcomes and comorbidities}

The control subjects were randomly selected as the patients without ESRD by matching study subjects according to age ( \pm 3 years), gender, and the year of admission at a 2:1 ratio from the same dataset. To investigate the risk of DVT, the patients started from the index date were followed until DVT (ICD-9-CM code 453.8), death, or withdrawal from the NHI occurred or the end of 2010. Comorbidities, including hypertension, diabetes, hyperlipidemia, cerebrovascular accident, congestive heart failure, atrial fibrillation, lower leg fracture or surgery, and cancer, were identified from 1 year before the index date according to ICD-9-CM codes from the outpatient and inpatient records.

\section{Statistical analysis}

Demographic and comorbidity variables were expressed as frequencies (percentages) or means ( \pm standard deviations, SDs). The continuous variables were distributed normally after normality test. The demographic characteristics were gender and age (stratification in the following age groups: $\leq 39,40-49,50-59,60-69$, and over 70 years). The incident rates of DVT were estimated using the total number of events divided by the total follow-up period (per 10,000 person-years).

Cox proportional hazards models were conducted to compare the risk of DVT between ESRD and non-ESRD groups. Joint effects with associated risk factors (age and comorbidities) for the risk of DVT in the ESRD group were also analyzed by Cox proportional hazards models. The covariate variables included gender and comorbidities. Proportionality of hazards assumption was tested and fulfilled before cox regression was conducted. Hazard ratios (HRs) and their 95\% confidence intervals (CIs) were estimated by Cox regression. Cumulative incidence functions of DVT for different groups were plotted and compared using competing risk methods [17]. All statistical 


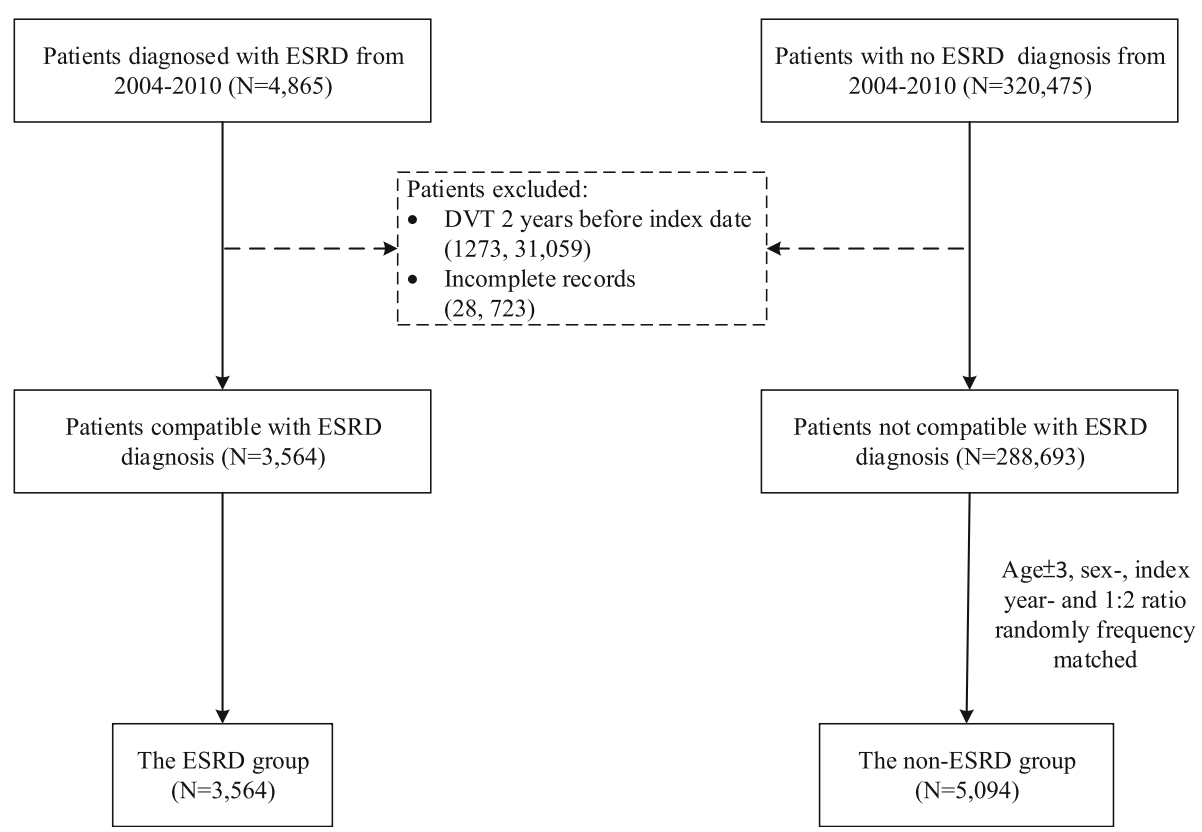

Fig. 1 Flowchart of subject enrollment

tests were two-sided, and a P-value of 0.05 was considered significant.

\section{Results}

\section{Patient characteristics}

The ESRD group comprised 3564 patients. The mean age was $62.76 \pm 14.69,35.7 \%$ of patients were aged $\geq 70$ years, and $49.4 \%$ of patients were male (Table 1 ). Similarly, the non-ESRD group had 5094 patients. The mean age was $61.84 \pm 15.00$ years, $33.1 \%$ of patients were aged $\geq 70$ years, and $49.1 \%$ were male. The distributions of age and gender among both groups were similar. Patients in the ESRD group had a significantly higher prevalence of comorbidities than the non-ESRD group in atrial fibrillation ( $1.8 \%$ vs. $0.5 \%)$, hypertension ( $56.8 \%$ vs. $26.9 \%)$, diabetes (39.4\% vs. $13.4 \%)$, hyperlipidemia (5.8\% vs. $1.2 \%$ ), cerebrovascular accident ( $11.1 \%$ vs $2.7 \%)$, congestive heart failure $(17.3 \%$ vs $1.3 \%)$, lower leg fracture or surgery $(1.2 \%$ vs $0.5 \%)$, and cancer ( $2.3 \%$ vs $1.9 \%$ ).

\section{Incidence of DVT}

In Table 2, the incident rate of DVT was substantially higher in the ESRD group than in the group without ESRD (20.9 vs. 1.46 per $10^{\wedge} 4$ person-years). The adjusted HR (aHR 13.92; 95\% CI 9.25-20.95) of DVT indicated that the risk of DVT for the ESRD patients was 13.92 times that of the non-ESRD patients. This relationship was further investigated to examine the associations among age, gender, and comorbidities. In the ESRD group, the DVT incidence rates stratified by age $(\leq 30,40-49$, $50-59,60-69$ and over 70 years) had corresponding aHRs
Table 1 Demographic characteristics and comorbidities in patients with and without ESRD

\begin{tabular}{lll}
\hline & Non-ESRD $(n=5094)$ & $\operatorname{ESRD}(n=3564)$ \\
\hline Age & & \\
$\leqq 39$ & $412\left(8.1^{\mathrm{a}}\right)$ & $265(7.4)$ \\
$40-49$ & $677(13.3)$ & $406(11.4)$ \\
$50-59$ & $1137(22.3)$ & $772(21.7)$ \\
$60-69$ & $1180(23.2)$ & $849(23.8)$ \\
$\geqq 70$ & $1688(33.1)$ & $1272(35.7)$ \\
Mean \pm SD & $61.84 \pm 15.00$ & $62.76 \pm 14.69$ \\
Gender & & \\
Male & $2499(49.1)$ & $1762(49.4)$ \\
Female & $2595(50.9)$ & $1802(50.6)$ \\
Deep vein thrombosis & $26(0.5)$ & $200(5.6)$ \\
Comorbidities & & $64(1.8)$ \\
Atrial fibrillation & $24(0.5)$ & $2024(56.8)$ \\
Hypertension & $1369(26.9)$ & $1404(39.4)$ \\
Diabetes & $684(13.4)$ & $208(5.8)$ \\
Hyperlipidemia & $63(1.2)$ & $394(11.1)$ \\
Cerebrovascular accident & $135(2.7)$ & $616(17.3)$ \\
Congestive heart failure & $65(1.3)$ & $44(1.2)$ \\
Lower leg fracture or surgery & $28(0.5)$ & $82(2.3)$ \\
Cancer & $99(1.9)$ & \\
\hline Abbrevatons: ESRD end-stage & & \\
\hline
\end{tabular}

Abbreviations: $E S R D$ end-stage renal disease, $S D$ standard deviation ${ }^{\text {apercentage }}$ 
Table 2 Incidence of DVT in patients with and without ESRD

\begin{tabular}{|c|c|c|c|c|c|c|c|c|}
\hline \multirow[t]{2}{*}{ Characteristics } & \multicolumn{3}{|c|}{ Non-ESRD $(n=5094)$} & \multicolumn{3}{|c|}{$\operatorname{ESRD}(n=3564)$} & \multirow[b]{2}{*}{ aHR $(95 \%$ Cl) } & \multirow[b]{2}{*}{$P$} \\
\hline & Event & TFP (PY) & $\mathrm{IR}$ & Event & TFP (PY) & $\mathbb{R}$ & & \\
\hline DVT & 26 & $18,112.37$ & 1.46 & 200 & 9552.35 & 20.94 & $13.92(9.25-20.95)$ & $<0.001$ \\
\hline \multicolumn{9}{|l|}{ Age } \\
\hline$\leqq 39$ & 2 & 1767.13 & 1.13 & 13 & 1004.83 & 12.94 & $10.99(2.48-48.73)$ & 0.002 \\
\hline $40-49$ & 1 & 2729.76 & 0.37 & 20 & 1477.39 & 13.54 & 36.76 (4.93-273.0) & $<0.001$ \\
\hline $50-59$ & 5 & 4334.96 & 1.15 & 47 & 2348.80 & 20.01 & $15.96(6.34-40.15)$ & $<0.001$ \\
\hline $60-69$ & 7 & 4303.83 & 1.63 & 58 & 2270.50 & 25.55 & 15.25 (6.95-33.46) & $<0.01$ \\
\hline$\geqq 70$ & 11 & 4976.70 & 2.21 & 62 & 2450.81 & 25.30 & $11.06(5.81-21.06)$ & $<0.01$ \\
\hline \multicolumn{9}{|l|}{ Gender } \\
\hline Male & 10 & 8833.28 & 1.13 & 91 & 4745.28 & 19.18 & 16.31 (8.49-31.36) & $<0.001$ \\
\hline Female & 16 & 9279.10 & 1.72 & 109 & 4807.06 & 22.67 & $12.46(7.37-21.08)$ & $<0.01$ \\
\hline \multicolumn{9}{|l|}{ Comorbidities } \\
\hline \multicolumn{9}{|l|}{ Hypertension } \\
\hline Yes & 8 & 4974.73 & 1.61 & 125 & 5714.18 & 21.88 & $13.21(6.46-27.02)$ & $<0.001$ \\
\hline No & 18 & $13,137.64$ & 1.37 & 75 & 3838.16 & 19.54 & $13.61(8.13-22.79)$ & $<0.001$ \\
\hline \multicolumn{9}{|l|}{ Diabetes } \\
\hline Yes & 4 & 2515.32 & 1.59 & 89 & 3607.59 & 24.67 & 15.01 (5.50-40.93) & $<0.01$ \\
\hline No & 22 & $15,597.06$ & 1.41 & 111 & 5944.75 & 18.67 & $12.72(8.05-20.10)$ & $<0.01$ \\
\hline \multicolumn{9}{|c|}{ Hyperlipidemia } \\
\hline Yes & 0 & 217.13 & 0 & 19 & 525.90 & 36.13 & $34.41(0.50-2985)$ & .102 \\
\hline No & 26 & $17,895.24$ & 1.45 & 181 & 9026.45 & 20.05 & $13.17(8.73-19.88)$ & $<0.01$ \\
\hline \multicolumn{9}{|c|}{ Cerebrovascular accident } \\
\hline Yes & 1 & 391.38 & 2.55 & 18 & 901.44 & 19.97 & $7.48(1.00-56.07)$ & 0.05 \\
\hline No & 25 & $17,720.00$ & 1.41 & 182 & 8650.90 & 21.04 & $14.29(9.40-21.71)$ & $<0.01$ \\
\hline \multicolumn{9}{|c|}{ Congestive heart failure } \\
\hline Yes & 0 & 184.50 & 0 & 30 & 1304.25 & 23.00 & $24.50(0.20-3057)$ & .194 \\
\hline No & 26 & $17,927.88$ & 1.45 & 170 & 8248.09 & 20.61 & $13.68(9.05-20.68)$ & $<0.01$ \\
\hline \multicolumn{9}{|c|}{ Lower leg fracture or surgery } \\
\hline Yes & 0 & 90.47 & 0 & 4 & 87.07 & 45.94 & $68.62(0.02-241,330)$ & .310 \\
\hline No & 26 & $18,021.90$ & 1.44 & 196 & 9465.28 & 20.70 & $13.71(9.10-20.65)$ & $<0.01$ \\
\hline \multicolumn{9}{|l|}{ Cancer } \\
\hline Yes & 1 & 281.68 & 3.55 & 3 & 153.36 & 19.56 & $4.40(0.46-42.45)$ & .201 \\
\hline No & 25 & $17,830.70$ & 1.40 & 197 & 9398.99 & 20.96 & $14.30(9.43-21.69)$ & $<0.01$ \\
\hline
\end{tabular}

Abbreviations: ESRD end-stage renal disease, DVT deep vein thrombosis, TFP total follow-up period, $P Y$ per 10,000 person-years, IR incident rate per 10,000 person-years, $a H R$ adjusted hazard ratio

that increased accordingly $(12.94,13.54,20.01,25.55$, and 25.30). Similarly, incidence rates that increased with age were found in the non-ESRD group. The Cox regression models showed that age, gender, and comorbidities were associated with higher risks of DVT in the ESRD groups than in the non-ESRD group (Table 2). For example, for the patients with hypertension, the adjusted HR of DVT in the ESRD group was 13.21 (95\% CI 6.46-27.02; $P<0.001$ ) times that of the non-ESRD group.

\section{DVT occurring with other risk factors}

The joint effects for DVT between ESRD and associated risk factors were investigated further. As shown in Table 3, the ESRD patients older than 50 years had a higher risk of DVT (aHR 1.65; 95\% CI 1.13-2.40; $P=0.01$ ) indicated that the risk of DVT for the ESRD patients over 50 years old was 1.65 times that of the younger ones. The association between comorbidities and DVT for ESRD patients was further investigated. Hyperlipidemia 
Table 3 Joint Effects for DVT between ESRD and Associated Risk Factors ( $n=3564, I R=5.6 \%$ )

\begin{tabular}{|c|c|c|c|c|c|}
\hline Variable & Number (\%) & Events & Rate & $\operatorname{aHR}(95 \% \mathrm{Cl})$ & $P$ \\
\hline Age $>=50$ years & $2893(81.20)$ & 167 & 0.06 & $1.65(1.13-2.40)$ & 0.01 \\
\hline Hypertension & $2024(56.80)$ & 125 & 0.06 & $1.10(0.83-1.47)$ & 0.52 \\
\hline Diabetes & $1404(39.39)$ & 89 & 0.06 & $1.26(0.95-1.67)$ & 0.11 \\
\hline Hyperlipidemia & $208(5.84)$ & 19 & 0.09 & $1.73(1.08-2.78)$ & 0.02 \\
\hline Cerebrovascular accident & $394(11.05)$ & 18 & 0.05 & $0.90(0.56-1.47)$ & 0.68 \\
\hline Congestive heart failure & $616(17.28)$ & 30 & 0.05 & $1.04(0.70-1.53)$ & 0.86 \\
\hline Atrial fibrillation & $64(1.80)$ & 2 & 0.03 & $0.77(0.19-3.10)$ & 0.71 \\
\hline Lower leg fracture or surgery & $44(1.23)$ & 4 & 0.09 & $2.04(0.76-5.48)$ & 0.16 \\
\hline Cancer & $82(2.30)$ & 3 & 0.04 & $0.89(0.28-2.78)$ & 0.84 \\
\hline$\geq 1$ comorbidity & $2547(71.46)$ & 151 & 0.06 & $1.14(0.83-1.58)$ & 0.43 \\
\hline$\geq 2$ comorbidities & $1509(42.34)$ & 88 & 0.06 & $1.13(0.86-1.50)$ & 0.39 \\
\hline$\geq 3$ comorbidities & 630 (17.68) & 43 & 0.07 & $1.45(1.03-2.03)$ & 0.03 \\
\hline
\end{tabular}

Abbreviations: ESRD end-stage renal disease, DVT deep vein thrombosis

was significantly associated with an increased risk of DVT (aHR 1.73; 95\% CI 1.08-2.78; $P=0.02$ ). ESRD patients with three or more comorbidities had a substantially higher risk of DVT (aHR 1.45; 95\% CI 1.03-2.03; $P=0.03)$.

\section{Cumulative incidence functions of DVT}

Cumulative incidence functions for the ESRD and nonESRD groups illustrating the occurrence of DVT over time (Fig. 2) showed differences between the two study groups. In Fig. 3, ESRD patients with hyperlipidemia had a higher risk of DVT than ESRD patients with no hyperlipidemia and patients without ESRD with hyperlipidemia. Similar patterns of higher risk of DVT were also found for ESRD patients older than 50 years (Fig. 4) and for patients with 3 or more comorbidities (Fig. 5).

\section{Discussion}

Previous studies examining DVT in patients with ESRD were case reports or case series or reported adverse outcomes in clinical trials [16, 18-21], and some of these patients with DVT had risk factors for DVT such as malignancy, postoperative state, or prolonged hospitalization. There is little evidence and no previous epidemiological studies showing that ESRD itself predisposes individuals to DVT. To our knowledge, this is the first study that used a national medical database to evaluate the epidemiology of DVT in patients with ESRD. In our study, the incidence of DVT was 20.94 per 10,000 person-years, and ESRD patients had a higher risk of DVT than patients without ESRD, with an aHR of 13.92 (95\% CI 9.25-20.95) in an Asian population. The incidence of DVT and pulmonary embolism was lower in Asian country compared with Western ones. Previous study showed ESRD is associated

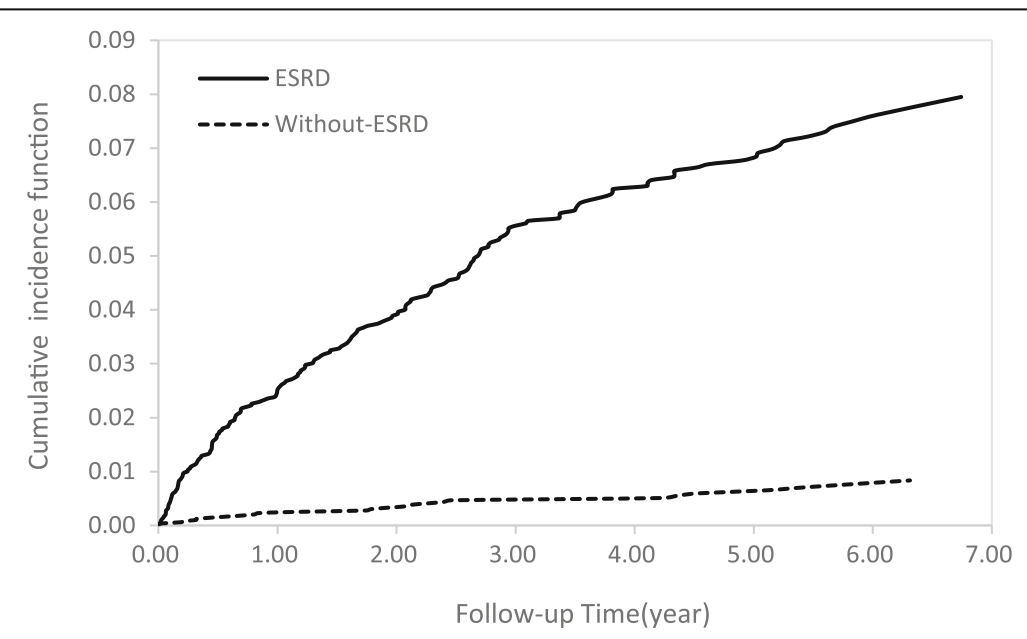

Fig. 2 Cumulative incidence function of DVT in patients with and without ESRD 


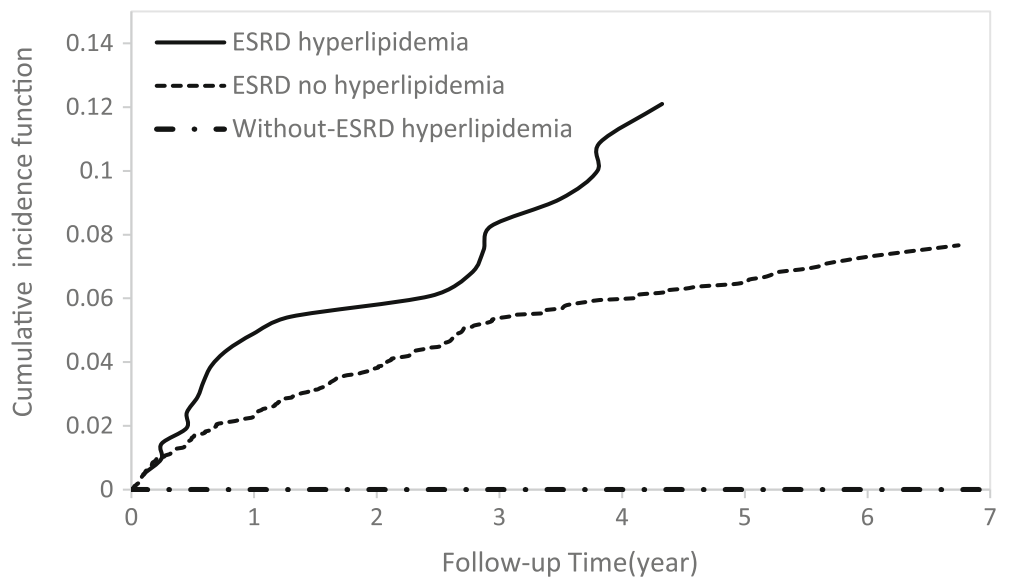

Fig. 3 Cumulative incidence function of DVT in patients with hyperlipidemia

with a 2.3-fold increased risk of DVT compared with the general population ${ }^{4}$. In Taiwan, ESRD patients was about 14-fold increased risk of DVT compared with non-ESRD patients. Treatment dyslipidemia and increased awareness in patients aged 50 or more are important for management DVT in Asian ESRD populations.

\section{ESRD and DVT}

The coagulation cascade is a complicated process that involves platelets, endothelial cells and coagulation factors. A bleeding tendency was frequently observed in clinical practice in ESRD patients, but thrombotic events, such as arteriovenous fistula thrombosis, peripheral arterial occlusive disease and DVT, were also noted in ESRD. According to the previous review data, the factors contributing to thrombotic events include hemodialyzer-induced platelet aggregation; elevated plasminogen activator inhibitor-1; increased Von Willebrand factor release; oxidative stress; increased homocysteine; fibrinogen; activity of factors VII,
VIII, and IX-XII; thromboplastin (tissue factor); fibrinopeptide $\mathrm{A}$; and reduced protein $\mathrm{C}$ [22]. In addition, there is a possibility that the clinical and demographic characteristics of the ESRD population continued to change, and treating anemia with erythropoietin or blood transfusion was associated with DVT [22].

According to previous literature and our previous study, DVT is less likely to occur among Asians than among Caucasians [23]. For example, the incidence of DVT in an Asian COPD population was 18.78 per 10,000 personyears, with an aHR of 1.38 (95\% CI 1.06-1.80) compared to a non-COPD population, after adjusting for age, sex, atrial fibrillation, hypertension, diabetes, hyperlipidemia, cerebrovascular accident, congestive heart failure, lower leg fracture and cancer, and the incidence was lower than that in the Caucasians population. This study showed that the incidence of DVT was higher in the ESRD population than in the non-ESRD population in Asians. To our knowledge, there is no recently published data regarding

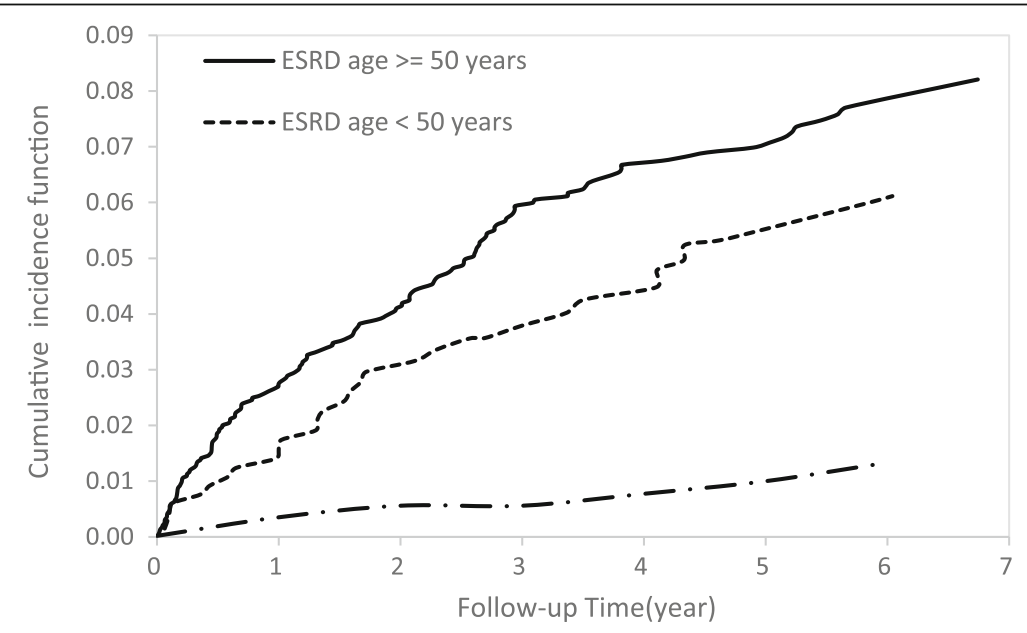

Fig. 4 Cumulative incidence function of DVT for patients aged over 50 years 


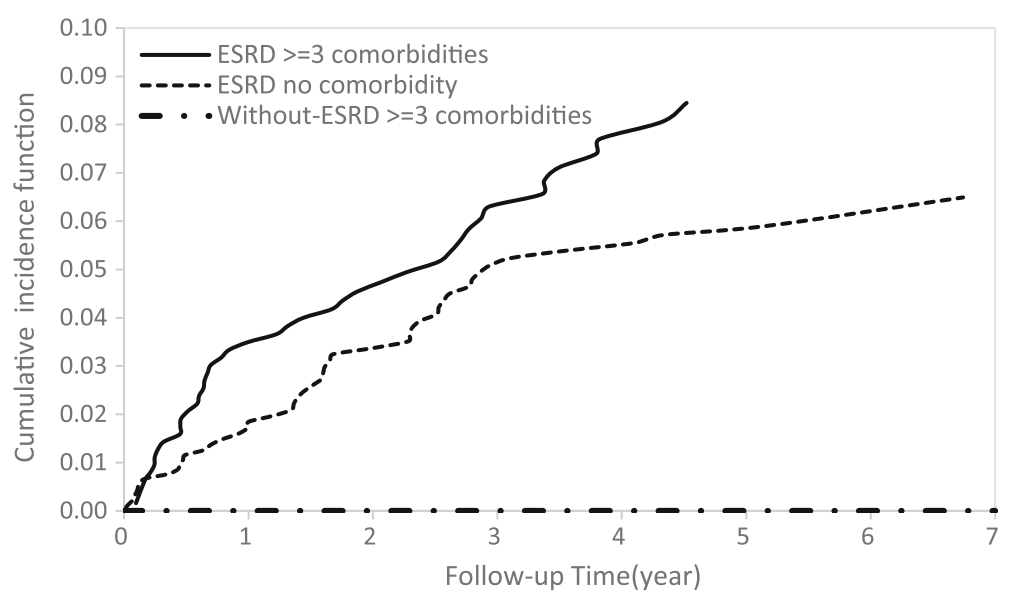

Fig. 5 Cumulative incidence function of DVT for patients with three or more comorbidities

the incidence of DVT in ESRD patients. According to previous studies utilizing administrative data in 2002, the incidence of venous thromboembolism, including DVT and pulmonary embolism, was 149.9 events per 100,000 dialysis patients [24]. The lower incidence rate may be related to data errors or incorrect coding [4].

\section{Age and DVT}

Age is a risk factor for DVT. According to previous studies, DVT was rare in young individuals, and the risk of DVT increased with age, or approximately $1 \%$ per year in the elderly [25]. In our study, we found that the incidence of DVT increased with age in the ESRD population. Compared to patients without ESRD, the highest incidence of DVT in the ESRD population occurred between 40 and 49 years of age, with an aHR of 36.76. ESRD placed patients aged between 40 and 49 years at greater risk for developing DVT. In addition, the aHR of DVT in the ESRD population was 1.65 for adults older than 50 years compared with adults younger than 50 years.

\section{Comorbidities and DVT}

Compared to patients without ESRD, patients with ESRD had some comorbidities that increased the risk of DVT, such as hypertension and diabetes. The well-known risk factors for the development of DVT, such as lower leg fracture or surgery and cancer, were not statistically significant for DVT because the number of cases of DVT in the non-ESRD population was zero or only one case. Further, the incidence of DVT was much lower in Asian populations than in Western populations. Future epidemiological studies on the incidence of DVT in patients with ESRD and their risk factors in Western populations may be needed.

In the ESRD population, being over 50 years old and having dyslipidemia were the only two risk factors for DVT in our study. Low incidence of DVT and a small case number for some comorbidities, such as atrial fibrillation, lower leg fracture or surgery, and cancer, may hamper these risk factors from reaching statistical significance. In our study, ESRD patients with more than three risk factors had a significantly increased risk of DVT.

\section{ESRD and acute kidney injury in DVT}

A population-based study enrolled 19,073 cases and authors found that CKD increased the risk of DVT [7]. A study in Taiwan that also used NHIRD [26] found that acute kidney injury increased the risk of DVT compared to that in patients with normal renal function. We not only studied the incidence of DVT in the ESRD population but also investigated the risk factors for DVT between ESRD and non-ESRD patients. In addition, we also surveyed the risk factors for DVT in the ESRD population. Future studies can clarify the association between DVT and renal function by delineating the risk of DVT at different stages compared to that of the normal population.

\section{Limitations}

The first of our limitations was the small number of cases of ESRD patients with comorbidities of lower leg fractures or surgery and cancer, which totaled only 87 and 153 person-years, respectively. Lower leg fracture or surgery and cancer are well-documented risk factors for DVT, but there is no significant correlation statistical significance in our study owing to a small number of cases. Second, one may doubt the accuracy of the database. In the NHI in Taiwan, patients with ESRD who required hemodialysis can apply for a catastrophic illness certificate and do not need to pay a co-payment for outpatient or inpatient care. This application will be formally reviewed by other physicians. The risk of incorrect coding is very low, and the validation of the NHIRD had been performed $[27,28]$. Otherwise, previous study from Canadian claim database has showed the diagnosis of DVT and pulmonary 
embolism in adult was sensitive by using ICD-9-CM [29]. Third, there are inherent limitations to this database. There is no smoking history, no body mass index data, no laboratory data and no image data. Otherwise, the potential information bias due to misclassification of DVT should be considered. In our study, we investigated the incidence of DVT in ESRD patients and their risk factors from a large nationwide database based on physician diagnosis in clinical practice. The comprehensive enrollment can represent real-world populations.

\section{Conclusions}

The incidence of DVT was higher in ESRD patients than in the non-ESRD population. Being older than 50 years and having dyslipidemia are risk factors for DVT among ESRD patients in Asian populations.

\section{Acknowledgements}

The authors express their sincere gratitude to Mr. Yi-Siou Chia (Department of Industrial Engineering and Management, National Yunlin University of Science and Technology) for his effort in organizing data and assisting statistical analysis.

\section{Availability of data and materials}

Data are available from the National Health Insurance Research Database (NHIRD) published by Taiwan National Health Insurance (NHI) Bureau. Due to legal restrictions imposed by the government of Taiwan in relation to the

"Personal Information Protection Act", data cannot be made publicly available.

\begin{abstract}
Authors' contributions
KML participated in the study conception and design, interpretation, and drafting of manuscript. HYL participated in the data analysis, interpretation, drafting of manuscript and extensive revision of the manuscript. Both authors had access to the data and can take responsibility for the integrity of the data and the accuracy of the data analysis. All authors read and approved the final manuscript.
\end{abstract}

\section{Ethics approval and consent to participate}

This study was approved by the Institutional Review Board (IRB) of the Chi Mei Medical Center, Taiwan (10609-E02).

\section{Consent for publication}

Not applicable.

\section{Competing interests}

The authors declare that they have no competing interests.

\section{Publisher's Note}

Springer Nature remains neutral with regard to jurisdictional claims in published maps and institutional affiliations.

\section{Author details}

${ }^{1}$ Department of Industrial Engineering and Management, National, Yunlin University of Science and Technology, Yun-Lin, Taiwan. ${ }^{2}$ Department of Internal Medicine, Chi Mei Medical Center, Chiali, Taiwan.

Received: 18 November 2017 Accepted: 20 July 2018

Published online: 16 August 2018

\section{References}

1. Anderson FA Jr, Wheeler HB, Goldberg RJ, et al. A population-based perspective of the hospital incidence and case-fatality rates of deep vein thrombosis and pulmonary embolism. The Worcester DVT study. Arch Intern Med. 1991;151:933-8.
2. Silverstein MD, Heit JA, Mohr DN, Petterson TM, O'Fallon WM, Melton LJ. Trends in the incidence of deep vein thrombosis and pulmonary embolism: a 25-year population-based study. Arch Intern Med. 1998;158:585-93.

3. Hansson PO, Welin L, Tibblin G, Eriksson H. Deep vein thrombosis and pulmonary embolism in the general population. Arch Intern Med. 1997:157:1665-70.

4. Wattanakit K, Cushman M. Chronic kidney disease and venous thromboembolism: epidemiology and mechanisms. Curr Opin Pulm Med. 2009;15:408-12.

5. Abbott KC, Cruess DF, Agodoa LY, Sawyers ES, Tveit DP. Early renal insufficiency and late venous thromboembolism after renal transplantation in the United States. Am J Kidney Dis. 2004;43:120-30.

6. Poli D, Zanazzi M, Antonucci E, Marcucci R, Rosati A, Bertoni E, Salvadori M, Liotta AA, Abbate R, Prisco D, Gensini GF. High rate of recurrence in renal transplant recipients after a first episode of venous thromboembolism. Transplantation. 2005:80:789-93.

7. Wattanakit K, Cushman M, Stehman-Breen C, Heckbert SR, Folsom AR. Chronic kidney disease increases risk for venous thromboembolism. J Am Soc Nephrol. 2008;19(1):135-40.

8. Keller C, Katz R, Cushman M, Fried LF, Shlipak M. Association of kidney function with inflammatory and procoagulant markers in a diverse cohort: a cross-sectional analysis from the multi-ethnic study of atherosclerosis (MESA). BMC Nephrol. 2008;9:9

9. Dobrowolski C, Clark EG, Sood MM. Venous thromboembolism in chronic kidney disease: epidemiology, the role of proteinuria, CKD severity and therapeutics. J Thromb Thrombolysis. 2017;43(2):241-7.

10. Königsbrügge $\mathrm{O}$, Lötsch F, Zielinski C, Pabinger I, Ay C. Chronic kidney disease in patients with cancer and its association with occurrence of venous thromboembolism and mortality. Thromb Res. 2014;134(1):44-9.

11. Kooiman J, den Exter PL, Cannegieter SC, le Cessie S, del Toro J, Sahuquillo JC, Pedrajas JM, Huisman MV. Impact of chronic kidney disease on the risk of clinical outcomes in patients with cancer-associated venous thromboembolism during anticoagulant treatment. J Thromb Haemost. 2013;11(11):1968-76.

12. Chertow GM, Mahaffey KW. Venous thromboembolism: yet another cardiovascular complication of chronic kidney disease? Circulation. 2012;126(16):1937-8.

13. Mahmoodi BK, Gansevoort RT, Næss IA, et al. Association of mild to moderate chronic kidney disease with venous thromboembolism: pooled analysis of five prospective general population cohorts. Circulation. 2012;126(16):1964-71.

14. Folsom AR, Lutsey PL, Astor BC, Wattanakit K, Heckbert SR, Cushman M. Atherosclerosis risk in communities study. Chronic kidney disease and venous thromboembolism: a prospective study. Nephrol Dial Transplant. 2010;25(10):3296-301.

15. Wiesholzer M, Kitzwögerer M, Harm F, et al. Prevalence of preterminal pulmonary thromboembolism among patients on maintenance hemodialysis treatment before and after introduction of recombinant erythropoietin. Am J Kidney Dis. 1999;33(4):702-8.

16. Casserly LF, Reddy SM, Dember LM. Venous thromboembolism in end-stage renal disease. Am J Kidney Dis. 2000;36(2):405-11.

17. Noordzij M, Leffondre K, van Stralen KJ, et al. When do we need competing risks methods for survival analysis in nephrology? Nephrol Dial Transplant. 2013;28:2670-7.

18. Maruyama H, Gejyo F, Hanano M, Arakawa M. Acquired type II protein C deficiency in a long-term hemodialysis patient. Nephron. 1994;66:348-50.

19. Mallamaci F, Zoccali C, Tripepi G, et al. Hyperhomocysteinemia predicts cardiovascular outcomes in hemodialysis patients. Kidney Int. 2002;61:609-14.

20. Wagner R, Mattes J, Traindl $\mathrm{O}$. Deep venous thrombosis in haemodialysis patients - a pointer to the presence of malignancy? Nephrol Dial Transplant. 1996;11:879-80.

21. Zoccali C, Bode-Boger S, Mallamaci F, Benedetto F, Tripepi G, Malatino L, Cataliotti A, Bellanuova I, Fermo I, Frolich J, Boger R. Plasma con- centration of asymmetrical dimethylarginine and mortality in patients with end-stage renal disease: a prospective study. Lancet. 2001:358:2113-7.

22. Casserly LF, Dember LM. Thrombosis in end-stage renal disease. Semin Dial. 2003:16(3):245-56.

23. Chen CY, Liao KM. The incidence of deep vein thrombosis in Asian patients with chronic obstructive pulmonary disease. Medicine (Baltimore). 2015;94(44):e1741.

24. Tveit DP, Hypolite IO, Hshieh P, et al. Chronic dialysis patients have high risk for pulmonary embolism. Am J Kidney Dis. 2002;39:1011-7. 
25. Engbers MJ, van Hylckama VA, Rosendaal FR. Venous thrombosis in the elderly: incidence, risk factors and risk groups. J Thromb Haemost. 2010;8(10):2105-12.

26. Kuo TH, Li HY, Lin SH. Acute kidney injury and risk of deep vein thrombosis and pulmonary embolism in Taiwan: a nationwide retrospective cohort study. Thromb Res. 2017;151:29-35.

27. Cheng CL, Lee CH, Chen PS, Li YH, Lin SJ, Yang YH. Validation of acute myocardial infarction cases in the national health insurance research database in Taiwan. J Epidemiol. 2014;24(6):500-7.

28. Cheng CL, Kao YH, Lin SJ, Lee CH, Lai ML. Validation of the National Health Insurance Research Database with ischemic stroke cases in Taiwan. Pharmacoepidemiol Drug Saf. 2011;20(3):236-42.

29. Tagalakis V, Kahn SR. Determining the test characteristics of claims-based diagnostic codes for the diagnosis of venous thromboembolism in a medical service claims database. Pharmacoepidemiol Drug Saf. 2011;20(3):304-7.

Ready to submit your research? Choose BMC and benefit from:

- fast, convenient online submission

- thorough peer review by experienced researchers in your field

- rapid publication on acceptance

- support for research data, including large and complex data types

- gold Open Access which fosters wider collaboration and increased citations

- maximum visibility for your research: over $100 \mathrm{M}$ website views per year

At $\mathrm{BMC}$, research is always in progress.

Learn more biomedcentral.com/submissions 